\title{
Vitrification preserves chromatin integrity, bioenergy potential and oxidative parameters in mouse embryos
}

\author{
Nicola A Martino ${ }^{1 *}$, Maria E Dell'Aquila ${ }^{1}$, Rosa A Cardone ${ }^{2}$, Bence Somoskoi ${ }^{3}$, Giovanni M Lacalandra ${ }^{1}$ \\ and Sandor Cseh $^{3}$
}

\begin{abstract}
Background: The aim of this study was to evaluate the effects of vitrification on morpho-functional parameters (blastomere/chromatin integrity and bioenergy/oxidative potential) of mouse preimplantation embryos.

Methods: In vivo produced mouse (4/16-cell, morulae and blastocyst-stage) embryos were randomly divided into vitrification and control groups. For vitrification, embryos were exposed to a 2-step loading of ethylene glycol and propylene glycol, before being placed in a small nylon loop and submerged into liquid nitrogen. After warming, the cryoprotectants were diluted by a 3-step procedure. Embryo morphology, chromatin integrity and energy/ oxidative status were compared between groups.
\end{abstract}

Results: Vitrification induced low grade blastomere cytofragmentation $(P<0.05)$ and low chromatin damage only in embryos at the morula stage $(P<0.001)$. Mitochondrial $(\mathrm{mt})$ distribution pattern was affected by vitrification only in early embryos $(P<0.001)$. Mitochondrial activity did not change upon vitrification in morula-stage embryos but it was reduced in blastocyst-stage embryos $(P<0.05)$. Intracellular ROS levels significantly increased in embryos at the morula and blastocyst stages $(\mathrm{P}<0.001)$. Colocalization of active mitochondria and ROS increased only in vitrified blastocysts.

Conclusions: In conclusion, this study elucidates the developmentally-related and mild effects of vitrification on morphology, nuclear and bioenergy/oxidative parameters of mouse embryos and demonstrates that vitrification is a suitable method for preserving predictive parameters of embryo ability to induce a full-term pregnancy.

\section{Background}

Vitrification is an alternative approach to freezing, which avoids the formation of ice crystals in the intracellular and extracellular space [1-3]. Vitrification is the solidification of a solution at low temperature, a process achieved by a combination of a high concentration of cryoprotectants (CPAs,4-8 M) and an extremely high cooling rate $[4,5]$.

Appropriate mitochondrial (mt) distribution and membrane potential in embryos are very important for developmental potential and for a variety of cellular activities, including ATP synthesis and specific cell functions $[6,7]$. However, there is little information available about the effects of vitrification on chromatin integrity, $\mathrm{mt}$

\footnotetext{
* Correspondence: nicmartino@libero.it

'Veterinary Clinics and Animal Productions Unit, Department of Emergency and Organ Trasplantation (DETO), University of Bari Aldo Moro, Valenzano, Bari, Italy

Full list of author information is available at the end of the article
}

dynamics/distribution and ROS production in oocytes/ embryos. Zhao et al. 2009 [8] reported that, in mouse two-pronuclear stage embryos, vitrification reduces the rate of $\mathrm{mt}$ ring formation around pronuclei, an event which may affect the syngamy of male and female pronuclei and subsequent embryo development. Shi et al. [9] reported that vitrification alters the $\mathrm{mt}$ distribution pattern in porcine MII oocytes.

It has been reported that oxidative stress (OS) may be an important mechanism underlying the toxic effects of cryopreservation procedures, which may then trigger the apoptosis cascade leading to a decrease in the survival and developmental rate of gametes and embryos after thawing [10-21]. Oxidative stress occurs if a disequilibrium takes place between reactive oxygen species (ROS) production and antioxidative capacity of the cell [14] and it has also been implicated in the etiology of some forms of female infertility [15]. Mitochondria represent

\section{Biomed Central}


the major source of ROS and they are produced in a stepwise process with a final reduction of $\mathrm{O}_{2}$ to $\mathrm{H}_{2} \mathrm{O}$ during oxidative phosphorylation, in particular at the level of complex I and III [16]. Under physiological conditions, ROS are neutralized by an elaborate defence system consisting of enzymes such as catalase, superoxide dismutase, glutathione peroxidase or reductase and numerous non enzymatic antioxidants such as vitamin $\mathrm{C}, \mathrm{E}, \mathrm{A}$, pyruvate, glutathione, ubiquinone, taurine and hypotaurine [17]. Thus, any perturbation in mt activity or in the activity of scavenger systems can lead to profound alterations in ROS production, OS induction, and mt cytochrome c release, which is an important step for apoptosis [18]. Embryos, as other aerobic cells, produce ATP and ROS by means of mt oxidative phosphorylation.

To our knowledge, the effects of vitrification on embryo morphology, chromatin integrity, mt distribution, energy status and ROS production in different developmental stages have not been documented to date. Therefore, the aim of the present study was to investigate the effects of vitrification on morpho-functional parameters of mouse embryos at different developmental stages. Blastomere morphology, expressed in terms of blastomere cytofragmentation; chromatin integrity, assessed as the rate of embryos showing low or high grade chromatin damage; mt distribution pattern, if either heterogeneous or homogeneous, indicating strong or weak energy condition, respectively; mt activity and intracellular levels of ROS, expressed as fluorescent intensities of specific probes, and mt/ROS colocalization, were examined. Our study could contribute to understanding whether, during embryo development, mt display dynamic tubular networks undergoing fission, fusion, organization in granules and tubules and intracellular movements as it has been demonstrated within the oocyte and whether any change in these events upon the use of cryopreservation procedure.

\section{Methods}

\section{Embryo recovery and in vitro culture}

Procedures with animals were performed following good veterinary practice for animal welfare according to Hungarian national laws in force. The protocol of the animal experiment was approved by the Ethical Committee of the Faculty of Veterinary Science Szent Istvan University of Budapest. Embryos were produced as reported by Klambauer et al., 2009 [19]. Briefly, eight weeks old CB6F1 female mice (Institute of Oncology, Animal Care Facility, Budapest, Hungary) were superovulated by 10 IU eCG i.p., followed by 10 IU hCG i.p. 48 h later, in order to induce the final maturation of the oocytes and ovulation. After the hCG injection the females were paired with males ( 1 female/male), then 20 to $24 \mathrm{~h}$ later the embryos were collected (Day 1). Until vitrification or analysis (fresh control embryos), embryos were cultured in G1 medium
(Vitrolife, Goteborg, Sweden) at $37.5^{\circ} \mathrm{C}$ with $6.5 \% \mathrm{CO}_{2}$ and maximal humidity in air for further 20 to 96 h. Only morphologically normal embryos, being in cleavage stage (4- to 16-cell) or at the morula or blastocyst stage, were randomly destined to either vitrification or fresh control groups.

\section{Vitrification}

Embryo vitrification was performed with the VitroLoop vitrification procedure as previously described [19] and unless otherwise specified, all materials were provided by Vitrolife. Briefly, embryos were exposed to a 2-step loading of the CPA solution, ethylene glycol (EG) and propylene glycol (PG), before being placed on a thin filmy layer formed from the vitrification solution in a small nylon loop, then they were rapidly submerged in liquid nitrogen $\left(\mathrm{LN}_{2}\right)$. Vitrification was carried out in RapidVit Cleave vitrification solutions (Vitrolife; solution 1: holding medium, solution 2: equilibration medium, and solution 3: vitrification medium) and embryos were manipulated in 4-well culture dish (Nunc Intermed, Roskilde, Denmark) held on a warming plate at $37^{\circ} \mathrm{C}$. The holding or basic solution is based on G-MOPS ${ }^{\mathrm{Tm}}$ and was supplemented with gentamycin and human serum albumin (HSA, $5 \mu \mathrm{g} / \mathrm{ml}$ ). Both the equilibration and the vitrification media are based on the holding/basic solution, but the equilibration medium was supplemented with $8 \% \mathrm{EG}+8 \% \mathrm{PG}$ and the vitrification solution was enriched with 16\% EG, 16\% PG, F (Ficoll, F-400, $10 \mathrm{mg} / \mathrm{ml}$ ) and $S$ (Sucrose, $0.65 \mathrm{~mol} / \mathrm{l}$ ). All manipulations of the embryos during their preparation for vitrification were carried out at $37^{\circ} \mathrm{C}$ (on a heated stage). Embryos were suspended from solution 1 into the equilibration medium (solution 2) for $2 \mathrm{~min}$. Thereafter, they were transferred and washed quickly in small drops of vitrification medium (solution 3). The cryoloop was dipped into the vitrification medium to create a thin filmy layer of the solution on the nylon loop where embryos (max 3 embryos) were quickly transferred from the vitrification medium. Within $30 \mathrm{sec}$ of suspension in the vitrification medium, the loop with the embryos was plunged into $\mathrm{LN}_{2}$. Embryos were warmed and rehydrated by a 3-step dilution of the CPA performed at $37^{\circ} \mathrm{C}$. At warming, the embryos were moved through a series of G-MOPS ${ }^{\text {тM }}$ solutions containing the $\mathrm{S}$ in decreasing concentrations (warming solution 1: $0.65 \mathrm{~mol} / \mathrm{l}$; $30 \mathrm{sec}$, warming solution 2: $0.25 \mathrm{~mol} / \mathrm{l}$; $1 \mathrm{~min}$, warming solution 3: $0.125 \mathrm{~mol} / \mathrm{l} ; 2 \mathrm{~min}$ and warming solution 4: $0.0 \mathrm{~mol} / \mathrm{l} ; 5 \mathrm{~min}$ ) (RapidWarm Cleave; Vitrolife, Goteborg, Sweden).

\section{Mitochondria and ROS staining}

Fresh and vitrified-warmed embryos underwent $\mathrm{mt}$ and ROS staining following the procedure by Ambruosi et al., 2011 and Martino et al., 2012 [20,21]. Embryos were 
washed three times in PBS with 3\% bovine serum albumin (BSA) and incubated for $30 \mathrm{~min}$ in the same medium containing $280 \mathrm{nM}$ MitoTracker Orange CMTM Ros (Molecular Probes M-7510, Oregon, USA) at $38.5^{\circ} \mathrm{C}$ under $5 \% \mathrm{CO}_{2}$ in air. The cell-permeant probe contains a thiolreactive chloromethyl moiety. Once the MitoTracker probe accumulates in the mitochondria, it can react with accessible thiol groups on peptides and proteins to form an aldehyde-fixable conjugate. This cell-permeant probe is readily sequestered only by actively respiring organelles depending on their oxidative activity [22,23]. After incubation with MitoTracker Orange CMTM Ros, embryos were washed three times in PBS with $0.3 \%$ BSA and incubated for $15 \mathrm{~min}$ in the same media containing $10 \mathrm{mM}$ 2,7'-dichlorodihydrofluorescein diacetate (DCDHF DA). The non-ionized DCDHF DA is membrane permeant and therefore is able to diffuse readily into cells. Once within the cell, the acetate groups are hydrolysed by intracellular esterase activity forming 2,7'-dichlorodihydrofluorescein (DCDHF) which is polar and thus trapped within the cell. DCHF fluoresces when it is oxidized by $\mathrm{H} 2 \mathrm{O} 2$ or lipid peroxides to yield 2,7'-dichlorofluorescein (DCF). The level of DCF produced within the cells is linearly related to that of peroxides present and thus its fluorescent emission provides a measure of the peroxide levels [24]. After incubation, embryos were washed three times in prewarmed PBS without BSA and fixed with $3.7 \%$ paraformaldehyde solution in PBS. All procedures after thawing/ warming were performed within 1 hour. Embryos were kept in fixative at $4^{\circ} \mathrm{C}$ for no longer than two to three days. The organelle-specificity of the mt probe was assessed, as reported by Valentini et al., 2010; [25], in control samples which were imaged after incubation in MitoTracker Orange and further incubation for $5 \mathrm{~min}$ in the presence of $5 \mathrm{mM}$ of the mt membrane potential (Delta Psi)-collapsing uncoupler carbonyl cyanide 3-chloro phenylhydrazone (CCCP; Molecular Probes), which inhibits $\mathrm{mt}$ respiratory activity thus reducing fluorescence intensity. Particular attention was paid to avoid sample exposure to the light during staining and fixing procedures in order to reduce photobleaching.

\section{Embryo morphology assessment}

Embryos at the different examined developmental stages were recovered from vials and their morphological appearance was assessed by evaluating blastomere cytofragmentation following the criteria described in previous studies in the mouse as well as in other species $[26,27]$. Embryos were examined at $400 \mathrm{x}$ magnification phase contrast microscopy and scored as either unfragmented (Grade 0), or with fragmentation graded into three categories as reported by Van Soom et al., 2003 and Han et al., 2005 [26,27]. Grade 1 fragmentation was defined as the presence of one or more cytofragments smaller than the size of a polar body and often clustered at one or both poles or in the crevice between blastomeres. Grade 2 fragmentation was defined as the presence of many more fragments, with total volume of fragments comprising an equivalent of less than one-half the volume of a blastomere. Grade 3 fragmentation was assigned to embryos with many large fragments, with the total volume of fragments being approximately onehalf of one blastomere or greater.

\section{Detection of the nuclear chromatin status}

To evaluate nuclear chromatin, embryos were stained with $2.5 \mu \mathrm{g} / \mathrm{ml}$ Hoechst 33258 in 3:1 (v/v) glycerol/PBS, mounted on microscope slides, covered with cover-up micro slides, sealed with nail polish and kept at $4^{\circ} \mathrm{C}$ in the dark until observation [20]. Nuclear chromatin status was observed under a Nikon Eclipse 600 fluorescent microscope equipped with B2A (346 nm excitation/ $460 \mathrm{~nm}$ emission) filter. Embryos were classified as normal (grade A) when the presence of a regular-shaped nucleus inside each blastomere was observed. The formation of micronuclei and lobulated nuclei was considered as signs of chromatin damage [28]. Embryos showing 0 to $20 \%$ affected blastomeres were classified as grade B and embryos with more than 20\% affected blastomeres were classified as grade $\mathrm{C}$.

\section{Mitochondrial distribution pattern and intracellular ROS localization}

For mt distribution pattern evaluation, embryos were observed at $600 \times$ magnification in oil immersion with a Nikon C1/TE2000-U laser scanning confocal microscope. A helium/neon laser ray at $543 \mathrm{~nm}$ and the G-2 A filter (551 nm exposure/576 nm emission) were used to observe the MitoTracker Orange CMTM Ros. An argon ions laser ray at $488 \mathrm{~nm}$ and the B-2 A filter (495 $\mathrm{nm}$ exposure/519 $\mathrm{nm}$ emission) were used to observe the DCF. Scanning was conducted with 25 optical series from the top to the bottom of the embryo with a step size of $0.45 \mu \mathrm{m}$ to allow three-dimensional distribution analysis. General criteria for $\mathrm{mt}$ pattern definition were adopted on the basis of previous studies in mouse and human oocytes and embryos $[7,8,29,30]$, as well as in oocytes of other species [31]. Thus, an homogeneous/ even distribution of small $\mathrm{mt}$ aggregates throughout the cytoplasm was considered as an indication of low energy cytoplasmic condition. Heterogeneous/uneven distribution of small and/or large mt aggregates indicated metabolically active cytoplasm. In particular, the accumulation of active $\mathrm{mt}$ in the peripheral cytoplasm (pericortical mt pattern) and/or around the nucleus (perinuclear and perinuclear/pericortical mt pattern, $\mathrm{P} / \mathrm{P}$ ) was considered as characteristic of healthy cytoplasmic condition. Embryos showing irregular distribution of large mt 
clusters unrelated to the specific cell compartments were classified as abnormal. To our knowledge, few studies have reported to date on intracellular ROS localization and levels in mouse embryos [32,33] and no studies on vitrified mouse embryos have been reported.

\section{Quantification of Mitotracker Orange CMTM Ros and DCF fluorescence intensity}

Measurements of fluorescence intensities were performed in embryos having either heterogeneous (perinuclear/ pericortical) or homogeneous (small aggregates) mt distribution pattern. Embryos showing abnormal mt distribution pattern were excluded from this analysis. In each individual embryo, the fluorescence intensity was measured at the equatorial plane, with the aid of the EZ-C1 Gold Version 3.70 software platform for Nikon C1 confocal microscope. A circle of an area (arbitrary value $=100$ in diameter) was drawn in order to measure only the cytoplasmic area. Fluorescence intensity encountered within the programmed scan area was recorded and plotted against the conventional pixel unit scale (0-255). Quantification analysis was performed only on embryos at the morula or blastocyst stage. In fact, due to their round shape, late stage embryos allow the software set-up for quantification analysis as reported for oocytes [21]. Parameters related to fluorescence intensity were maintained at constant values for all evaluations. In detail, images were taken under fixed scanning conditions with respect to laser energy, signal detection (gain) and pinhole size.

\section{Mitochondria/ROS colocalization analysis}

Colocalization analysis of $\mathrm{mt}$ and ROS was performed by using the EZ-C1 Gold Version 3.70 software. For each channel, the same threshold, set to the zero value, was used for the data set. Degree of colocalization was reported as a Pearson's correlation coefficient quantifying the overlap degree between MitoTracker and DCDHF DA fluorescence signals $[21,34]$.

\section{Developmental ability of vitrified embryos}

In order to demonstrate whether vitrified embryos retain the developmental ability, a group of vitrified embryos were warmed and cultured in vitro as described above. Their developmental ability was compared with that of non vitrified control embryos. Moreover, the possible toxic effect of the cryoprotectant mixture was assessed, by comparing the developmental rate of non vitrified embryos exposed to cryoprotectants (non-vitrified/exposed) with that of non vitrified/non exposed embryos.

\section{Statistical analysis}

For each examined embryo developmental stage, embryo morphology (the rates of embryos showing cytoplasmic fragmentation and chromatin damage) and the rates of embryos showing the different $m$ t distribution patterns and ROS intracellular localization were compared between the fresh control and vitrified-warmed embryos by Chi square-analysis with the Yates correction for continuity. Fisher's exact test was used when a value of less than 5 was expected in any cell. Mean values (mean $\pm \mathrm{SD}$ ) of $\mathrm{mt}$ and ROS fluorescence intensities, expressed as arbitrary density units (ADU) and mt/ROS colocalization, expressed as Pearson's correlation coefficient, were compared by the Student's t-test. Differences with $\mathrm{P}<0.05$ were considered statistically significant.

\section{Results}

Two hundred and sixty-seven mouse embryos, 99 of which at the early stages of development (including 4/8-cell, $\mathrm{n}=42$ and 8/16-cell, $\mathrm{n}=57$ ), 122 at the morula stage (including 16/32-cell, $n=76$ and $>32$ cell, $n=46$ ) and 46 at the blastocyst stage, were randomly divided into vitrified ( $n=118$ embryos) and control ( $n=149$ embryos) groups.

\section{Vitrification increases low grade embryo blastomere cytofragmentation}

The overall proportion of fragmented embryos (grade $1+$ grade 2) was increased by vitrification ( $26 \%$ vs $5 \%$, for vitrified-warmed vs control embryos, respectively; $\mathrm{P}<0.05$ ). However, most of fragmented embryos were found to be of low grade, i.e. of grade 1 ( $24 \%$ and $5 \%$ grade 1 fragmented embryos in vitrified and control groups, respectively; $\mathrm{P}<0.05)$ and very few embryos were found to be of intermediate grade, i.e. of grade $2(2 \%$ and $0 \%$ grade 2 fragmented embryos in vitrified and control groups, respectively; NS). No high grade fragmentation (grade 3) was found in either group.

\section{Vitrification increases low level embryo chromatin damage}

Vitrification induced low level nuclear chromatin damage (26\% vs $5 \%$ in vitrified and control embryos, respectively; $\mathrm{P}<0.05$; Figure 1, panel A). Chromatin damage increased with embryo development and became statistically significant in embryos at the morula stage $(P<0.01)$. On the contrary, chromatin integrity was preserved in embryos at the blastocyst stage. Figure 1 (panel A) shows the overall (grade $\mathrm{B}+$ grade $\mathrm{C}$ ) proportion of embryos with damaged chromatin observed after vitrification (black bars), grouped according to their developmental stage, and compared with controls (white bars). In Additional file 1: Figure $\mathrm{S} 1$, the proportions of embryos showing grade $\mathrm{B}$ (0 to 20\%; panel A) or grade C (>20\%; panel B) chromatin damage are detailed. As depicted in panel A, the proportions of grade B affected embryos were significantly higher after vitrification (24\%) compared with controls $(5 \%, \mathrm{P}<0.05)$ while vitrification had no effect on the rates 


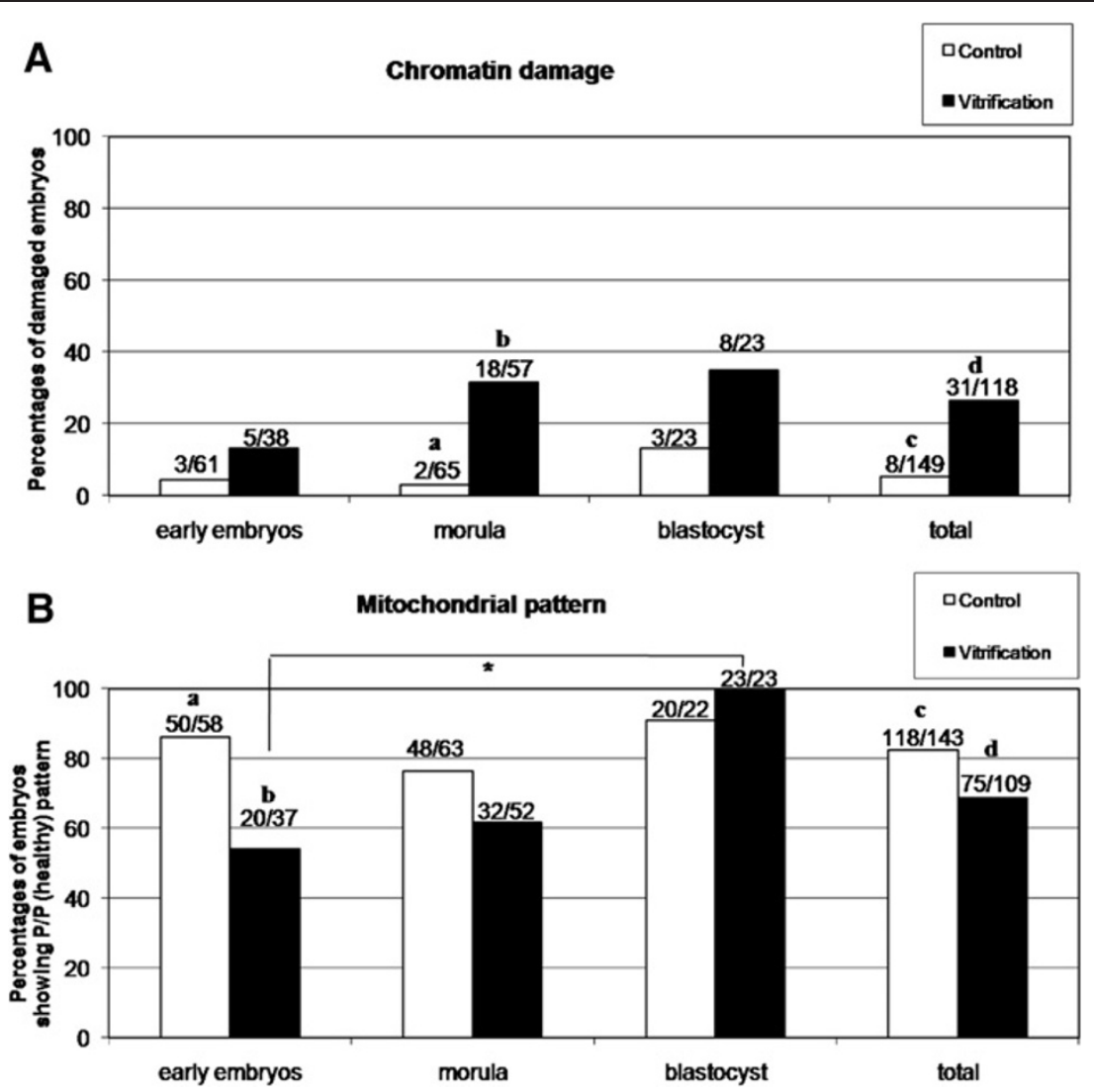

Figure 1 Effects of vitrification on chromatin integrity and mitochondrial distribution pattern of mouse 4/16-cell, morula and blastocyst stage embryos. Panel A: overall percentages of embryos (grade B + grade C) with damaged chromatin observed after vitrification (black bars), grouped according to their developmental stage, and compared with controls (white bars). In detail, in embryos at the morula stage, vitrification increased the rate of embryos showing chromatin damage whereas it had no effect in early embryos and in blastocysts. Chi square test: within each stage: $a, b P<0.001 ; c, d P<0.05$. Panel B:Percentages of embryos showing the $P / P$ mt pattern observed after vitrification (black bars), grouped according to examined developmental stages, and compared with controls (white bars). In detail, in embryos at the 4/16-cell stage, vitrification reduced the rate of embryos showing P/P $\mathrm{mt}$ pattern whereas it had no effect in embryos at the morula and blastocyst stages. Chi square test: $a, b P<0.001 ; c, d P<0.05 ;{ }^{*} P<0.001$. Numbers of analyzed oocytes per group are indicated on the top of each histogram.

of embryos showing grade $\mathrm{C}$ chromatin damage (3/118; 2.5\%). In Figure 1 panel $\mathrm{A}$ and in the Additional file 1, values plotted on the y axis are the proportion of embryos with chromatin damage, thus complementary percentages are referred to normal (grade A) embryos.

\section{Vitrification preserves $\mathrm{mt}$ distribution pattern and ROS localization in mouse embryos}

In Figure 1 (panel B), the percentages of embryos showing the $\mathrm{P} / \mathrm{P}$ (healthy) $\mathrm{mt}$ pattern observed after vitrification (black bars), grouped according to their developmental stage, and compared with controls (white bars), are reported. Apparently, vitrification reduced the ratio of embryos showing $\mathrm{P} / \mathrm{P} \mathrm{mt}$ pattern compared to controls (overall data: $\mathrm{P}<0.05$ ). However, the developmentallyrelated data showed that vitrification significantly reduced the ratio of embryos showing the $\mathrm{P} / \mathrm{P}$ mt pattern only at early stages $(\mathrm{P}<0.001)$.
In Figure 2, representative photomicrographs of embryo blastomere cytoplasmic shape and texture, as observed after staining and fixing procedures (lane 1) and nuclear chromatin (lane 2) of vitrified-warmed embryos at different developmental stages (rows B, D and F) and their non-vitrified control counterparts (rows $\mathrm{A}, \mathrm{C}$ and $\mathrm{E}$ ), are shown. In addition, Figure 2 shows representative photomicrographs of heterogeneous and homogeneous $\mathrm{mt}$ distribution pattern (lane 3) with corresponding intracellular ROS localization (lane 4) and mt/ROS merge (lane 5) in vitrified-warmed and control embryos at the 4/16-cell, morula and blastocyst stages. In control fresh embryos (Figure 2, rows A, C, E), MitoTracker signals were detected in all blastomeres in the form of continuous rings around the nuclei and clusters of mitochondria at the cortex (heterogeneous, perinuclear/pericortical, $\mathrm{P} / \mathrm{P} \mathrm{mt}$ pattern), which has been reported in previous studies as an indication of healthy embryos (Zhao et al., 2009 for 2 pronuclear mouse embryos; [8]). Intracellular ROS 


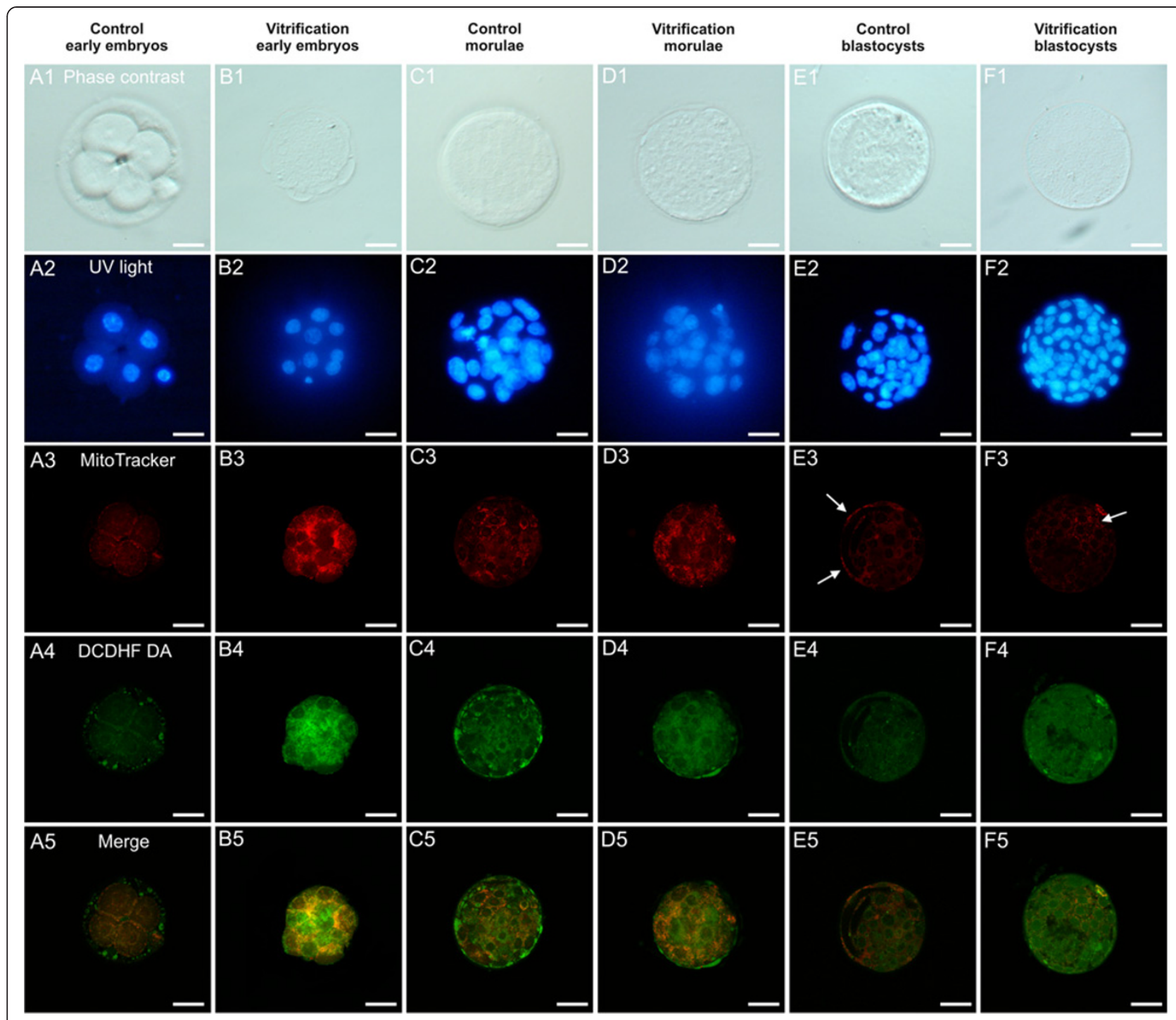

Figure 2 Photomicrographs of fresh and vitrified/warmed mouse embryos at early (4/16-cell), morula and blastocyst stages of development as assessed for their nuclear chromatin and bioenergy/oxidative potential. For each embryo, corresponding bright-field (phase contrast; lane 1), UV light (lane 2) and confocal images showing mt distribution pattern (lane 3), ROS localization (lane 4) and mt/ROS merge (lane 5), are shown. Representative photomicrographs of control non-vitrified and vitrified/warmed embryos at the 4- (row A) and 8-cell (row B), morula (rows C and D) and blastocyst stages (rows E and F), are shown. Nuclear chromatin was stained with Hoechst 33258.MitoTracker Orange CMTM Rosand DCDH FDA were used to label mitochondria and ROS, respectively. In control fresh early embryos and in morulae, in all blastomeres, there were detectable MitoTracker signals in the form of continuous rings around the nuclei and clusters of mitochondria at the cortex, namely perinuclear/pericorticalmt pattern (heterogeneous, healthy P/P mt pattern) (A3, C3). Vitrified early embryos(B3) showed a uniform/ diffused (homogeneous) mt distribution pattern throughout the blastomere cytoplasm. In embryos at the morula and blastocyst stage, the mt pattern was apparently not affected by vitrification (D3 vs C3 and F3 vs E3). A higher number of red fluorescent spots is evident on the trophoectoderma (white arrows) compared with the inner cell mass, indicating differences in mt number/cell between these two embryo lineages and higher mt/number and aggregate formation in the trophoectoderma compared with ICM. This feature can be observed in embryos of both groups, thus it was not influenced by vitrification. ROS intracellular localization (lane 4) corresponded to the distribution pattern of mitochondria. In fresh control embryos, apart areas/sites of mt/ROS overlapping (merge, lane 5), intracellular ROS appeared diffused throughout the cytoplasm (A4, C4, E4). In vitrified (B4, D4 and F4) embryos, diffused MitoTracker and DCDH FDA labelling were evident throughout the cytoplasm. Scale bar represents $20 \mu \mathrm{m}$. 

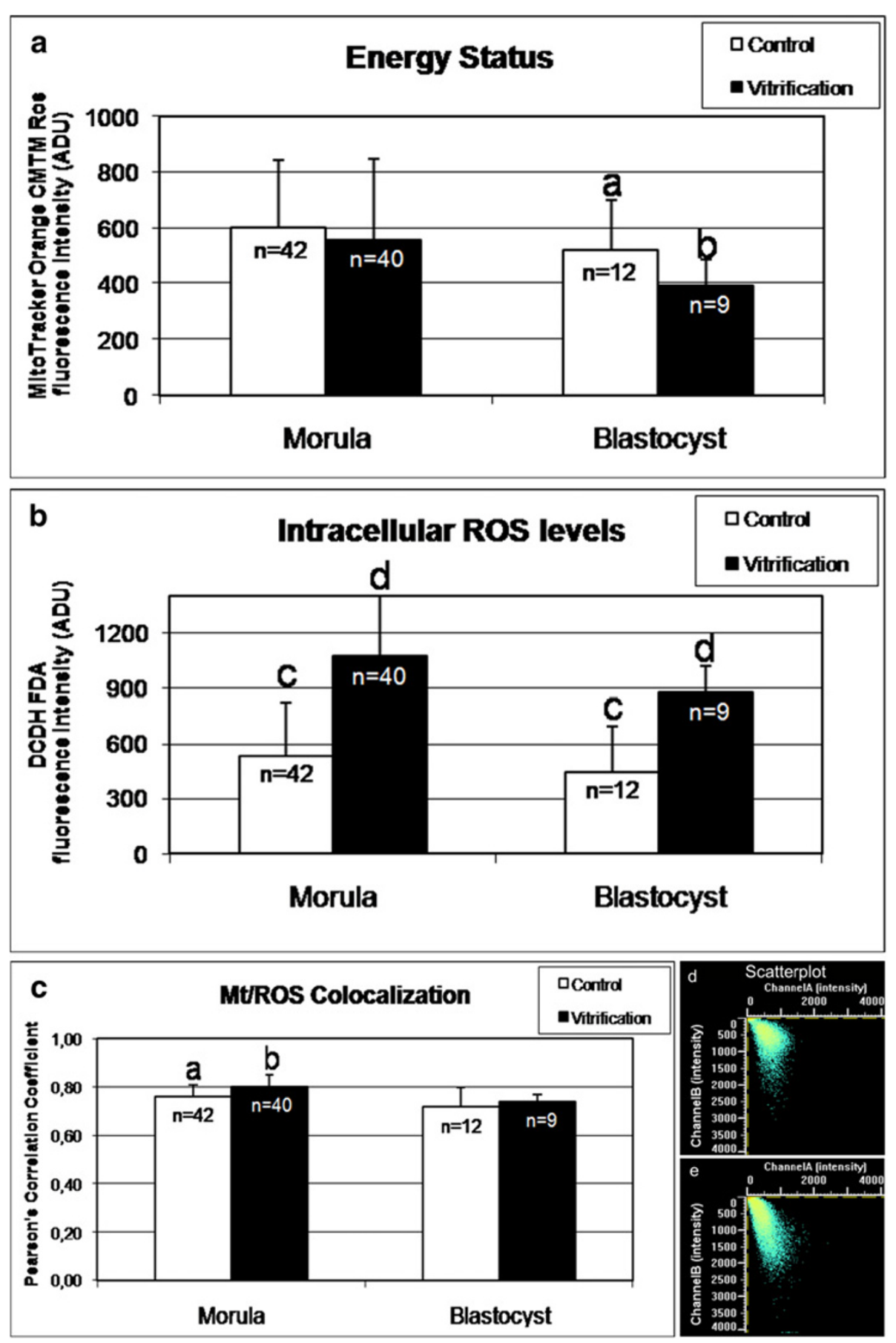

Figure 3 Effects of vitrification on mitochondrial activity, intracellular ROS levels and mt/ROS colocalization in single mouse morulae and blastocysts. In each group, energy status and ROS intracellular levels are expressed as mean \pm SD of Mitotracker Orange CMTM Ros (panel a) and DCF (panel b) fluorescence intensity of individual embryos in arbitrary densitometric units (ADU). In each group, mt/ROS colocalization is expressed as mean $\pm S D$ of Pearson's correlation coefficient of individual embryos (panel c). Representative mt/ROS colocalization scatterplots graph of a fresh (panel d) and a vitrified (panel e) blastocyst are shown. In embryos at the morula stage, mt activity did not change after vitrification whereas in embryos at the blastocyst stage, $m t$ activity was significantly reduced $(P<0.05)$. ROS levels significantly increased in vitrified embryos at morulaand blastocyst stages $(\mathrm{P}<0.001)$. Mt/ROS colocalizationsignificantly increased in vitrifiedmorulae. Numbers of analyzed embryos per group are indicated on the top of each histogram. Student's t-Test: a,b $P<0.05 ; c, d P<0.001$. 
Table 1 In vitro developmental ability of vitrified mouse embryo

\begin{tabular}{lcc}
\hline Groups & $\begin{array}{c}\mathbf{N}^{\circ}(\%) \text { of cleavage stage } \\
\text { embryos }\end{array}$ & $\begin{array}{c}\mathbf{N}^{\circ}(\%) \text { of embryos developed in vitro to } \\
\text { expanded blastocysts }\end{array}$ \\
\hline Vitrified embryos & 202 & $180(81.9)$ \\
Non-vitrified embryos exposed to cryoprotectants & 75 & $65(86.6)$ \\
Non-vitrified/non-exposed embryos (controls) & 82 & $75(91.4)$ \\
\hline
\end{tabular}

Chi-square test: NS.

appeared diffused throughout the cytoplasm in embryonic blastomeres at any stage of development and in both groups (vitrification and controls) apart areas/sites of $\mathrm{mt} /$ ROS overlapping (Figure 2, lane 4). In Figure 2, in embryos at the blastocyst stage, a higher number of red fluorescent spots was found on the trophoectoderm compared with the inner cell mass (ICM; E3 and F3), indicating differences in mt number or activation status per cell, between these two embryo lineages. Possibly, an higher $\mathrm{mt}$ number and/or aggregate formation of active mitochondria is found in the trophoectoderm compared with ICM. This feature was observed in all groups (23\% and 22\% for control and vitrified embryos: NS) and thus was not influenced by vitrification procedure.

\section{Vitrification alters $\mathrm{mt}$ activity and ROS levels but preserves $\mathrm{mt} / \mathrm{ROS}$ colocalization in mouse preimplantation embryos}

Mitochondrial activity and intracellular ROS levels were evaluated at the equatorial plane of embryos which were vitrified at the morula or blastocyst stage, having a round shape and thus allowing the confocal quantification software set-up in areas describing continuous surfaces as for oocyte analysis [21]. Mitochondrial activity did not change upon vitrification in embryos at the morula stage, whereas it was reduced in embryos at the blastocyst stage (Figure 3, Panel a; $\mathrm{P}<0.05$ ). Intracellular ROS levels significantly increased in both morula and blastocyst stage embryos (Figure 3, Panel b; $\mathrm{P}<0.001$ ). Mitochondria/ROS colocalization significantly increased $(\mathrm{P}<0.05)$ in vitrified versus control embryos in embryos at the morula stage whereas it did not change in embryos at the blastocyst stage (Figure 3, Panel c). In Figure 3, representative samples of $\mathrm{mt} / \mathrm{ROS}$ colocalization scatterplots for a fresh (Panel d) and a vitrified (Panel e) blastocyst, are also shown.

\section{Vitrification does not affect mouse embryo developmental ability}

The results of in vitro embryo culture obtained in the different treatment groups (vitrified, non-vitrified but exposed and non-vitrified/non-exposed to cryoprotectants control embryos) are presented in Table 1. A total of 229 cleavage stage embryos were vitrified/warmed, and out of them 11 were lost $(11 / 229 ; 4.8 \%)$. From the remaining 218 embryos, 202 survived vitrification (202/218; 92.7\%) and
180 developed further to expanded blastocysts during in vitro culture $(180 / 202 ; 89.1 \%)$. In the group of embryos non-vitrified but exposed, $86.6 \%$ of the embryos developed to expanded blastocysts (65/75). In the group of control embryos (non-vitrified/non-exposed), $91.4 \%$ of the embryos developed to expanded blastocysts (75/82).

\section{Discussion}

Vitrification slightly affected embryo morphology by increasing the percentage of embryos showing low grade (less than 20\%) blastomere cytofragmentation. Embryo fragmentation is clinically considered as an indicator of reduced embryo viability and developmental ability, reduced number of high quality embryos available for establishing pregnancies, thus as a relevant problem for assisted reproduction outcome. In fresh embryos, blastomere fragmentation has been reported to be associated to apoptosis $[24,35,36]$ or to apoptosis and necrosis [37]. A recent study [36] revealed novel early transcription mechanisms by which maternal genotype affects cytofragmentation by alterating regular cytoskeletal functions. In the present study, in which embryos were examined no more than two hours after warming (including staining procedures), these mechanisms could be only hypothesized. Thus, it can be concluded that vitrification increases only mild cytofragmentation, thus allowing the preservation of embryo blastomeres integrity, and not reducing the number of embryos available for transfer.

Vitrification increased low level chromatin damage $\mathrm{P}<0.05)$. Moreover, only intermediate stages of development were affected. Although not many studies have been published so far, our observations are in line with results of previous studies demonstrating, with different methods, lower damaging effects of vitrification compared with slow freezing on embryo chromatin integrity and function. Vutyavanich et al., 2009 [38] reported significantly higher average number of nuclei in blastocysts derived from embryos vitrified at the 2-cell stage, and cultured in vitro, compared with those obtained after slow freezing. Other studies examined the effects of cryopreservation methods on DNA integrity and stability, as assessed by TUNEL test. Tsang and Chow, 2010 [39] reported significant reduction of DNA integrity after both procedures. AbdelHafez et al., 2011 [40], reported higher DNA damage extent in vitrified blastocyst versus in early cleavage stage vitrified embryos, may 
be attributable to differences in blastocoel shrinkage after exposure to vitrification solutions. To our knowledge, this is the first study including all developmental stages and comparing stage-specific effects of vitrification on chromatin integrity of mouse embryos.

Bioenergy/oxidative stress analysis can be performed with several molecular and biochemical methods [41]. Global assessment strategies, such as OMICS technologies, such as Transcriptomics, Proteomics, Metabolomics, are becoming increasingly valuable in this area of investigation [42]. For the specific purpose of assisted reproduction, particularly for research on oocytes and embryos, confocal imaging allows global qualitative and quantitative evaluation of bioenergy/redox parameters in individual samples, also enabling the localization and quantification of functional aberration. As for parameters of cytoplasmic maturity, reduced percentages of embryos showing $\mathrm{mt} \mathrm{P} / \mathrm{P}$ distribution pattern were observed only at early stages of development (4/16-cell stage, $\mathrm{P}<0.001$ ) compared with fresh control embryos. Moreover, at any stage of development the rate of vitrified embryos showing $\mathrm{P} / \mathrm{P}$ pattern never dropped below $50 \%$ and in total samples, more than $70 \%$ of embryos retained this $\mathrm{mt}$ pattern. The qualitative analysis of fluorescence, indicative of $\mathrm{mt}$ activity, conducted on fresh 4/16-cell stage embryos demonstrated that: 1) mt localization was perinuclear and pericortical, 2) there were blastomeres with intense $\mathrm{mt}$ activity while others with almost no activity, and 3) spots of intense mt activity were evident at the level of blastomere cell junctions. This observation is in agreement with those reported by Van Blerkom, 2009 [43], who showed that $\mathrm{mt}$ activity can influence or can be influenced by intercellular contacts. Our observations are in agreement with a previous study by Zhao et al., 2009 [8] who reported that in fresh 2PN mouse embryos stained with JC-1, red colored mitochondria (high $\Delta \psi$ ) were distributed primarily around pronuclei and along the cell membrane whereas in vitrified-warmed $2 \mathrm{PN}$ embryos, red $\mathrm{mt}$ were greatly diminished with green $\mathrm{mt}$ (low $\Delta \psi$ ) evenly distributed throughout the cytoplasm. At the same time, these authors found that the proportion of fresh $2 \mathrm{PN}$ embryos with normal aggregation of high $\Delta \psi \mathrm{mt}(84 \%)$ was significantly higher than that of vitrified $2 \mathrm{PN}$ embryos (27\%). Observed altered mt distribution could be due to modifications of cytoskeletal elements which have been reported to be involved in cellular movement on a rapid timescale of these organelles [18], other cytoplasmic components, such as endoplasmic reticulum [18], or to modifications of specific proteins involved in $\mathrm{mt}$ anchoring to cytoskeletal microfilaments or microtubules [44]. Previous studies in the mouse reported no significant differences after vitrification in microfilament distribution in zygotes, 2 cell embryos, morulae and blastocysts [39] and in microtubule formation in 2PN embryos [8].
In embryos at the morula and blastocyst stage, the qualitative analysis showed that $\mathrm{mt}$ compartimentalization, which at these stages is indicative of developmental stagedependent acquisition of blastomeres cytoplasmic maturity, was not affected by vitrification. This observation could be related to major cryotolerance but also to a greater difficulty in visualizing $\mathrm{mt}$ distribution modification in embryos at these stages due to reduced blastomere cytoplasmic size. Qualitative analysis also showed that mt activity of trophoectodermal cells in blastocyst stage embryos is more intense than that observed in the cells of the inner cell mass and that blastomeres showing strong mt activity were located nearby the blastocoelic cavity. This observation is in agreement with those reported by Van Blerkom, 2011, [7] who showed that the maintenance of the blastocoele and its rapid recovery after collapse and hatching phase, are morphodynamics activities that require huge production of ATP from the cells of the trophoectoderma. Instead, cells in the inner cell mass, which are not involved in these activities, appear to be metabolically quiescent in these developmental phases. Quantitative analysis of ATP production in mouse blastocysts showed that approximately $80 \%$ of ATP produced by the embryo is from the trophoectoderma and that the number of $\mathrm{mt}$ observed by confocal laser microscopy are located well below in the inner cell mass [7].

Quantification analysis in the present study was performed in embryos at the morula and blastocyst stage. A statistically significant reduction of MitoTracker fluorescence intensity was found in vitrified blastocysts compared with their fresh counterparts, indicating significant reduction of $\mathrm{mt}$ activity at this stage of development. In embryos at the morula stage, vitrification had no effects on mt activity. More interestingly, both in embryos at the morula and blastocyst stage, significant increase of DCF fluorescence intensity, indicative of an excess production of ROS, was found after vitrification. This finding could be interpreted considering that the conditions used for the vitrification method could have resulted in the onset of oxidative stress condition. This observation could be consistent with the reported upregulation of genes involved in the mechanisms of oxidative stress (Hsp70, MnSOD, CuSOD) in mouse vitrified embryos [39]. Increased oxidative stress in vitrified/warmed embryos in the present study, kept in vitro no more than two hours after warming, could be an initial effect, as suggested by Tsang and Chow, (2010; [39]) who reported that stress-related gene expression dropped down to normal levels within 7 hours after warming. Colocalization of intracellular free radicals (ROS) and actively respiring $\mathrm{mt}$ has been reported as indicative of higher ATP turnover resulting from a more intense mt activity and thus indicative of healthy cell conditions in ovine in vivo matured ovulated metaphase 
II oocytes [21] and in hepatocytes. By this analysis, it came out that $\mathrm{mt} / \mathrm{ROS}$ colocalization was not affected by vitrification in blastocyst stage embryos and it was increased in embryos at the morula stage, may be due to increased ROS generation. To our knowledge, this is the first study reporting $\mathrm{mt} / \mathrm{ROS}$ colocalization, objectively expressed as Pearson's correlation coefficient, for the comparison between fresh and vitrified mouse embryos.

Taken together our data allow to confirm that morula and blastocyst are good stages from the standpoint of embryo viability after vitrification. In vitrified morulae, only low level chromatin damage was found and bioenergy/redox parameters were positively affected. Infact, $\mathrm{mt}$ pattern was not affected and increased oxidative activity and consequent increased mt/ROS colocalization were found. In vitrified blastocysts, neither nuclear chromatin nor mt pattern were affected; a significant reduction of $\mathrm{mt}$ activity was found but ADU absolute values remained at consistent levels $(395.1 \pm 89.3$ versus $522.4 \pm 176.8$ for vitrified vs fresh, respectively), indicating that vitrified embryos retained/kept a good/substantial part of mt activity. As well, in vitrified blastocysts increased respiratory/ oxidative activity was found, as observed by increased ROS generation.

\section{Conclusions}

The vitrification technology only slightly affects embryo morphology, chromatin integrity and energy/oxidative status in a developmentally-related manner. This embryo cryopreservation methods is of great scientific and clinical interest and application in both human and animal assisted reproduction. Global assessment strategies for embryo quality evaluation, such as confocal 3D imaging, can significantly contribute to the improvement of routine use crypreservation protocols, as well as to identify appropriate conditions to preserve nuclear and cytoplasmic integrity and competence to ensure proper embryonic development in the uterine environment and that the pregnancy could come to term. This study shows for the first time the joint assessment of mitochondrial activity and levels of ROS in mouse embryos in relation to their developmental stage and the application of a vitrification procedure.

\section{Additional file}

Additional file 1: Figure S1. Percentages of embryos showing either 0 to $20 \%$ (Panel A: grade B) or $>20 \%$ (Panel B: grade C) chromatin damage. Numbers of analyzed embryos per group are indicated on the top of each histogram. Chi square test: within each stage: $a, b P<0.001 ; c, d$ $P<0.05$; between stages: $\left(^{*}\right) P<0.05$.

\section{Competing interests}

The authors declare that they have no competing interests.

\section{Authors' contributions}

Conceived and designed the experiments: SC, MED, NAM, BS, GML. Performed the experiments: NAM, BS, SC, MED. Analyzed the data: NAM, SC, MED, BS, RAC. Contributed reagents/materials/analysis tools: SC, MED, GML. Wrote the paper: NAM, SC, MED, BS, RAC, GML. Experiments supervision and critical reading of the manuscript: MED, NAM, RAC, SC. All authors read and approved the final manuscript.

\section{Acknowledgments}

The research was funded by the Hungarian Academy of Sciences and supported by the Academic Center of Excellence: "Comparative Genomics: Genes Involved in Physiopathological Processes in the Biomedical and Agricultural Fields" University of Bari Aldo Moro, Italy, and by the projects COFIN PRIN MIUR 2008, University of Bari Aldo Moro, Italy cod. 2008YTYNKE_001, and Finanziamenti d'Ateneo 2010, University of Bari Aldo Moro, Italy, cod. ORBA10YTAK (both to M.E.D.). The study was also sponsored by the ONEV project "Omica e Nanotecnologie applicate agli Esseri Viventi per la diagnosi di malattie; MIUR PONa3_00134 - Avviso n254/R\&C 18/05/ 2011"and by the TÁMOP-4.2.2.B-10/1 and TÁMOP-4.2.1.B-11/2/KMR-2011-0003 projects. The funders had no role in study design, data collection and analysis, decision to publish, or preparation of the manuscript.

\section{Author details}

${ }^{1}$ Veterinary Clinics and Animal Productions Unit, Department of Emergency and Organ Trasplantation (DETO), University of Bari Aldo Moro, Valenzano, Bari, Italy. ${ }^{2}$ Department of Bioscience, Biotechnology and Biopharmaceutics, University of Bari, Aldo Moro, Bari, Italy. ${ }^{3}$ Department and Clinic of Obstetrics and Reproduction, Szent Istvan University, Budapest, Hungary.

Received: 31 October 2012 Accepted: 17 March 2013

Published: 3 April 2013

\section{References}

1. Fuller B, Paynter S, Watson P: Cryopreservation of human gametes and embryos. In Life in the frozen state. Edited by Fuller B, Lane N, Benson E. Boca Raton: CRC Press; 2004:505-541.

2. Rall WF, Fahy GM: Ice-free cryopreservation of mouse embryos at -196 degree C by vitrification. Nature 1985, 313:573-575.

3. Vajta G: Vitrification of oocytes and embryos of domestic animals. Anim Reprod Sci 2000, 60-61:357-364.

4. Rall WF: Factors affecting the survival of mouse embryos cryopreserved by vitrification. Cryobiology 1987, 24:387-402.

5. Liebermann J, Nawroth F, Isachenko V, Isachenko E, Rahimi G, Tucker MJ: Potential importance of vitrification in reproductive medicine. Biol Reprod 2002, 67:1671-1680.

6. Van Blerkom J, Davis P, Alexander S: Differential mitochondrial distribution in human pronuclear embryos leads to disproportionate inheritance between blastomeres: Relationship to microtubular organization, ATP content and competence. Hum Reprod 2000, 15:2621-2633.

7. Van Blerkom J: Mitochondrial function in the human oocyte and embryo and their role in developmental competence. Mitochondrion 2011, 11:797-813.

8. Zhao XM, Fu XW, Hou YP, Yan CL, Suo L, Wang YP, Zhu HB, Dinnyés A, Zhu SE: Effect of vitrification on mitochondrial distribution and membrane potential in mouse two pronuclear (2-PN) embryos. Mol Reprod Dev 2009, 76:1056-1063.

9. Shi LY, Jin HF, Kim JG, Kumar M, Balasubramanian S, Choe SY, Rho GJ: Ultra-structural changes and developmental potential of porcine oocytes following vitrification. Anim Reprod Sci 2007, 100:128-140.

10. Bilodeau JF, Chatterjee S, Sirard MA, Gagnon C: Levels of antioxidant defenses are decreased in bovine spermatozoa after a cycle of freezing and thawing. Mol Reprod Dev 2000, 55:282-288.

11. Ahn HJ, Sohn IP, Kwon HC, Jo DH, Park YD, Min CK: Characteristics of the cell membrane fluidity, actin fibers, and mitochondrial dysfunctions of frozenthawed two-cell mouse embryos. Mol Reprod Dev 2002, 61:466-476.

12. Somfai T, Ozawa M, Noguchi J, Kaneko H, Kuriani Karja NW, Farhudin M, Dinnyés A, Nagai T, Kikuchi K: Developmental competence of in vitrofertilized porcine oocytes after in vitro maturation and solid surface vitrification: effect of cryopreservation on oocyte antioxidative system and cell cycle stage. Cryobiology 2007, 55:115-126. 
13. Tatone C, Di Emidio G, Vento M, Ciriminna R, Artini PG: Cryopreservation and oxidative stress in reproductive cells. Gynecol Endocrinol 2010, 26:563-567

14. Finkel T, Holbrook NJ: Oxidants, oxidative stress and the biology of ageing. Nature 2000, 408:239-247.

15. Agarwal A, Allamaneni SS: Role of free radicals in female reproductive diseases and assisted reproduction. Reprod Biomed Online 2004, 9:338-347.

16. Cadenas E, Davies KJ: Mitochondrial free radical generation, oxidative stress, and aging. Free Radic Biol Med 2000, 29:222-230.

17. Winyard PG, Moody CJ, Jacob C: Oxidative activation of antioxidant defence. Trends Biochem Sci 2005, 30:453-461.

18. Brookes PS, Yoon Y, Robotham JL, Anders MW, Sheu SS: Calcium, ATP, and ROS: a mitochondrial love-hate triangle. Am J Physiol Cell Physiol 2004, 287:817-833.

19. Klambauer P, Keresztes Z, Kanyo K, Varga E, Kriston R, Vass N, Jávor A, Konc J, Solti L, Cseh S: Vitrification of cleavage stage mouse embryos by the cryoloop procedure. Acta Vet Hung 2009, 57(3):399-410.

20. Ambruosi B, Filioli Uranio M, Sardanelli AM, Pocar P, Martino NA, Paternoster MS, Amati F, Dell'Aquila ME: In vitro acute exposure to DEHP affects oocyte meiotic maturation, energy and oxidative stress parameters in a large animal model. PLoS One 2011, 6(11):e27452.

21. Martino NA, Lacalandra GM, Filioli Uranio M, Ambruosi B, Caira M, Silvestre F, Pizzi F, Desantis S, Accogli G, Dell'Aquila ME: Oocyte mitochondrial bioenergy potential and oxidative stress: within-/between-subject, in vivo vs IVM and age-related variations in a sheep model. Fertil Steril 2012, 97(3):720-728.

22. Poot M, Zhang YZ, Kramer JA, Wells KS, Jones LJ, Hanzel DK, Lugade AG, Singer VL, Haugland RP: Analysis of mitochondrial morphology and function with novel fixable fluorescent stains. J Histochem Cytochem 1996, 44:1363-1372.

23. Torner H, Brussow KP, Alm H, Ratky J, Pohland R, Tuchscherer A, Kanitz W: Mitochondrial aggregation patterns and activity in porcine oocytes and apoptosis in surrounding cumulus cells depends on the stage of preovulatory maturation. Theriogenology 2004, 61:1675-1689.

24. Yang HW, Hwang KJ, Kwon HC, Kim HS, Choi K, Oh KS: Detection of reactive oxygen species (ROS) and apoptosis in human fragmented embryos. Hum Reprod 1998, 13:998-1002.

25. Valentini L, lorga Al, De Santis T, Ambruosi B, Reynaud K, Chastant-Maillard S, Guaricci AC, Caira M, Dell'Aquila ME: Mitochondrial distribution patterns in canine oocytes as related to the reproductive cycle stage. Anim Reprod Sci 2010, 117:166-177.

26. Van Soom A: Assessment of mammalian embryo quality: what can we learn from embryo morphology. Reprod BioMed Online 2003, 7:104-110.

27. Han Z, Chung YG, Gao S, Latham KE: Maternal factors controlling blastomere fragmentation /in early mouse embryos. Biol Reprod 2005, 72:612-618.

28. Liu J, Tang S, Xu W, Wang Y, Yin B, Zhang Y: Detrimental effects of antibiotics on mouse embryos in chromatin integrity, apoptosis and expression of zygotically activated genes. Zygote 2010, 19:137-145.

29. Van Blerkom J, Davis P, Mathwig V, Alexander S: Domains of high-polarized and low-polarized mitochondria may occur in mouse and human oocytes and early embryos. Hum Reprod 2002, 17(2):393-406.

30. Van Blerkom J: High-polarized mitochondria $\left(\Delta \Psi_{\mathrm{m}} \mathrm{HIGH}\right)$ are spatially polarized in human oocytes and early embryos in stable subplasmalemmal domains: developmental significance and the concept of vanguard mitochondria. Reprod BioMed Online 2006, 13(2):246-254.

31. Ambruosi B, Lacalandra GM, lorga Al, De Santis T, Mugnier S, Matarrese R, Goudet G, Dell'aquila ME: Cytoplasmic lipid droplets and mitochondrial distribution in equine oocytes: implications on oocyte maturation, fertilization and developmental competence after ICSI. Theriogenology 2009, 71:1093-1104.

32. Zhang C, Liu C, Li D, Yao N, Yuan X, Yu A, Lu C, Ma X: Intracellular redox imbalance and extracellular amino acid metabolic abnormality contribute to arsenic-induced developmental retardation in mouse preimplantation embryos. J Cell Physiol 2010, 222(2):444-455.

33. Kawamura $Y$, Uchijima $Y$, Horike $N$, Tonami K, Nishiyama K, Amano T, Asano T, Kurihara $Y$, Kurihara $\mathrm{H}$ : Sirt3 protects in vitro-fertilized mouse preimplantation embryos against oxidative stress-induced p53-mediated developmental arrest. J Clin Invest 2010, 2;120(8):2817-2828.

34. Zinchuk V, Grossenbacher-Zinchuc O: Recent advances in quantitative colocalization analysis:focus on neuroscience. Prog Histochem Cytochem 2009, 44:125-172.
35. Jurisicova A, Varmuza S, Casper R: Programmed cell death and human embryo fragmentation. Mol Hum Reprod 1996, 2:93-98.

36. Han Z, Mtango NR, Zhong Z, Vassena R, Latham KE: Early transcription from the maternal genome controlling blastomere integrity in mouse two-cell stage embryos. Am J Physiol 2010, 298:1235-1244

37. Chi HJ, Koo JJ, Choi SY, Jeong HJ, Roh Sl: Fragmentation of embryos is associated with both necrosis and apoptosis. Fertil \& Steril 2011, 96:187-192.

38. Vutyavanich T, Sreshthaputra O, Piromlertamorn W, Nunta S: Closed-system solid surface vitrification versus slow programmable freezing of mouse 2-cell embryos. J Assist Reprod Genet 2009, 26:285-290.

39. Tsang WH, Chow KL: Cryopreservation of Mammalian Embryos: Advancement of Putting Life on Hold. Birth Defects Res 2010, 90:163-175.

40. AbdelHafez F, Xu J, Goldberg J, Desai N: Vitrification in open and closed carriers at different cell stages: assessment of embryo survival, development, DNA integrity and stability during vapor phase storage for transport. BMC Biotechnol 2011, 11:1-10.

41. Piccoli C, Sardanelli A, Scrima R, Ripoli M, Quarato G, D'Aprile A, Bellomo F, Scacco S, De Michele G, Filla A, luso A, Boffoli D, Capitanio N, Papa S: Mitochondrial respiratory dysfunction in familiar parkinsonism associated with PINK1 mutation. Neurochem Res 2008, 33(12):2565-2574.

42. Dell'Aquila ME, Cho YS, Martino NA, Filioli Uranio M, Rutigliano L, Hinrichs K: OMICS for the identification of biomarkers for oocyte competence, with special reference to the mare as a prospective model for human reproductive medicine. In Meiosis - Molecular Mechanism and Cytogenetic Diversity. Edited by InTech: 2012. ISBN 978-953-51-0118-5.

43. Van Blerkom J: Mitochondria in early mammalian development. Semin Cell Dev Biol 2009, 20:354-364.

44. Perkins GA, Tjong J, Brown JM, Poquiz PH, Scott RT, Kolson DR, Ellisman MH, Spirou GA: The micro-architecture of mitochondria at active zones: electron tomography reveals novel anchoring scaffolds and cristae structured for high-rate metabolism. J Neurosci 2010, 20;30(3):1015-1026.

doi:10.1186/1477-7827-11-27

Cite this article as: Martino et al: Vitrification preserves chromatin integrity, bioenergy potential and oxidative parameters in mouse embryos. Reproductive Biology and Endocrinology 2013 11:27.

\section{Submit your next manuscript to BioMed Central and take full advantage of:}

- Convenient online submission

- Thorough peer review

- No space constraints or color figure charges

- Immediate publication on acceptance

- Inclusion in PubMed, CAS, Scopus and Google Scholar

- Research which is freely available for redistribution 